\title{
BLADE MANUFACTURING TOLERANCES DEFINITION FOR A MISTUNED INDUSTRIAL BLADED DISK
}

\section{Evangéline CAPIEZ-LERNOUT}

\author{
Laboratory of Engineering Mechanics \\ University of Marne-La-Vallée \\ 5 , Boulevard Descartes \\ 77455, Marne-La-Vallée, France \\ Email: capiez@univ-mlv.fr
}

\author{
Christian SOIZE
}

Laboratory of Engineering Mechanics

University of Marne-La-Vallée

5, Boulevard Descartes

77455, Marne-La-Vallée, France Email: soize@univ-mlv.fr
Jean-Pierre LOMBARD

Snecma Moteurs

Site de Villaroche

77550, Moissy Cramayel, France

Email: jean-pierre.lombard@snecma.fr

\section{Christian DUPONT}

Snecma Moteurs

Site de Villaroche

77550, Moissy Cramayel, France

Email: christian.dupont@snecma.fr

\section{Eric SEINTURIER}

Snecma Moteurs

Site de Villaroche

77550, Moissy Cramayel, France

Email: eric.seinturier@snecma.fr

\begin{abstract}
This paper deals with the characterization of the blade manufacturing geometric tolerances in order to get a given level of amplification in the forced response of a mistuned bladed-disk. It is devoted to an industrial application in order to validate the theory previously developed [1] and in order to show that this theory is suited to any industrial bladed-disks. It should be noted that the development of an adapted methodology for solving the inverse problem, in order to characterize the manufacturing tolerances, is an important challenge for industries in this area. Let us recall that this theory is based on the use of a nonparametric probabilistic model of random uncertainties in the blade [2]. The dispersion parameters controlling the nonparametric model are estimated as a function of the geometric tolerances. Such an identification is carried out in a computational context by using the numerical Monte Carlo simulation and by using the reduced model method presented in [3]. The industrial application is devoted to the mistuning analysis of a 22 blades wide chord fan stage. Centrifugal stiffening due to rotational effects is also included. The results obtained validate the efficiency and the reliability of the method on three dimensional bladed disks.
\end{abstract}

\section{NOMENCLATURE}

$n$

$N$

$n_{b}$

$n_{d}$

$n_{i}^{d}$

$n_{i}$

$n_{\Sigma}$

$N_{b}$

$N_{d}$

$[A]^{T}$

$[A]^{*}$

$\operatorname{tr}[A]$

$\|[A]\|_{F}$

$\mathcal{E}$

$\mathcal{P}(X>x)$
Number of DOF of the bladed disk

Number of blades

Number of generalized coordinates for a blade Number of disk modes per circumferential wave number

Number of internal DOF of the disk Number of internal DOF of a blade Number of DOF on the coupling interface of the blade

Number of generalized coordinates for the blades equal to $n_{b} \times N$

Number of generalized coordinates for the disk equal to $n_{d} \times N$

Transpose of matrix $[A]$

Transconjuguate of matrix $[A]$

Trace of matrix $[A]$

Frobenius norm of matrix $[A]$ such that

$\|[A]\|_{F}=\operatorname{tr}\left([A][A]^{T}\right)^{1 / 2}$

Mathematical expectation

Probability that random variable $X$ be greater than a given level $x$ 


\begin{tabular}{|c|c|}
\hline $\mathbb{M}_{n, p}(\mathbb{R})$ & Set of all the $n \times p$ real matrices \\
\hline$M_{n}(\mathbb{R})$ & Set of all the $n \times n$ real matrices \\
\hline $\mathbb{M}_{n}^{+}(\mathbb{R})$ & $\begin{array}{l}\text { Set of all the positive-definite symmetric } n \times n \\
\text { real matrices }\end{array}$ \\
\hline$\delta_{i j}$ & $\begin{array}{l}\text { Kronecker symbol such that } \delta_{i j}=1 \text { if } i=j \text { and } \\
\delta_{i j}=0 \text { if } i \neq j\end{array}$ \\
\hline $\mathbb{B}$ & Frequency band of analysis \\
\hline$\Omega$ & Rotation velocity \\
\hline
\end{tabular}

\section{INTRODUCTION}

It is well known that the description of a bladed disk using its cyclic symmetry properties $[4,5]$ is not sufficient to predict accurately its dynamic forced response. In such a mechanical system, there are random uncertainties on the geometrical parameters, on the mechanical parameters related to the constitutive equations, on the boundary conditions, which are due to the manufacturing process of the blade. The blade mistuning terminology is then introduced because the blades of a bladed disk are slightly different from one to another one. Such a mistuning is not without consequence when analyzing the forced response of the bladed disk. Indeed, it is observed that the vibratory energy of the mistuned bladed disk is localized inducing large dynamic amplification factor $[6,7,8]$. Various researches have been carried out (see for instance $[9,10,11,12]$ for lumped parameters models, [13, 14, 15, 16, 17] for reduced order models) in order to understand and to control this phenomenon. This paper concerns a method which could be used for defining the specification of the blade manufacturing tolerances in order to have a given amplification factor due to the mistuning in a probabilistic context.

An exact parametric probabilistic approach should consist in setting up experimental means in order to construct a complete probabilistic model of all the parameters related to the random geometry of the blade. Then functions mapping the domain of uncertain parameters into the mass, damping and stiffness finite element matrices should be constructed. Then, efficient reduced order models $[15,17]$ should be used in order to analyze the forced response statistics of the bladed disk. It should be noted that such a parametric probabilistic approach would require a large number of uncertain parameters to be experimentally identified in particular for modeling the stochastic fields representing the geometrical random uncertainties.

In this paper, an alternative approach based on the use of the nonparametric probabilistic model of random uncertainties developed for linear and nonlinear elastodynamics $[18,19,20]$ is proposed. The theory concerning this inverse problem related to the definition of the blade manufacturing tolerances has been previously developed and validated for a simple numerical case [1]. Let us recall that the main steps of such an approach are based on (1) the construction of a mean reduced matrix model for each blade because the probability model related to the nonparametric approach is implemented from reduced matrices; (2) the construction of the probability model by using the maximum entropy principle under constraints defined by the available information; three scalar parameters allowing the dispersion of the random mass, damping and stiffness matrices to be controlled constitute the data input of the nonparametric approach; (3) the identification procedure of these dispersion parameters with respect to the physical parameters which describe the random geometry of the blades. The objective of this paper is the application of the theory previously developed to a realistic case consisting of an industrial bladed disk.

It should be noted that the mistuning is completely controlled by the probabilistic nonparametric model of the blade uncertainties. This nonparametric model is itself completely controlled by two scalar dispersion parameters $\delta_{M}$ and $\delta_{K}$ related to the mass and the stiffness operators of the blades. The proposed estimator of these dispersion parameters with respect to the blade geometry uncertainties is weakly sensitive to the choice of the probability model used for generating the blade random geometry. Consequently, it is important to note that the probabilistic model of the blade random geometry is not directly used for predicting the mistuning but only for estimating the dispersion parameters $\delta_{M}$ and $\delta_{K}$.

In a first part, a mean reduced matrix model both adapted to the nonparametric approach and to industrial numerical computations is briefly recalled. The theoretical results concerning the inverse problem are then adapted to the industrial bladed disk. Finally, the last section is devoted to a large finite element model of an industrial fan stage. A convergence analysis is performed in order to verify the choice of the reduced model dimension required for the stochastic analysis. Furthermore, a random geometry of the blade is defined in order to carry out the identification process of the dispersion parameters. Then the probability distribution of the random dynamic magnification factor is calculated as a function of the tolerance parameters.

\section{MEAN REDUCED MATRIX MODEL}

The structure considered is a three dimensional rotating fan stage modeled by a bladed disk with $N$ blades. The bladed disk is rotating around a fixed axis with the constant velocity $\Omega$ and is submitted to external forces. The vibration analysis is carried out in the rotating frame related to the reference configuration in the low-frequency band $\mathbb{B}$. The centrifugal terms are taken into account in the stiffness terms. Gyroscopic coupling due to rotating motion is not taken into account. All the blades are different from one to another due to manufacturing tolerances. Although the level of uncertainty is homogeneous for the blades, the random uncertainties are statistically independent from one to another blade. The construction of a mean reduced matrix model for each blade is thus required in order to model the random uncertainties with the nonparametric probabilistic approach. In this paper, the mean model of the structure is reduced by the substructuring method introduced by Benfield and Hruda [21]. The efficiency of this method has been proved for mistuned industrial bladed disks [17, 3] where each blade constitutes a branch component of the disk substructure. Each blade is reduced by using the Craig and Bampton method [22]. A reduced model of the disk with loaded coupling interface is constructed by modal 
analysis. In order to connect the substructures, the displacement of each blade on the coupling interface is projected on the disk modes by using the continuity of displacements at the coupling interface. The matrix equation related to the mean finite element model of the bladed disk is written as

$$
\left(-\omega^{2}[\underline{\mathbb{M}}]+i \omega[\underline{\mathbb{D}}]+[\underline{\mathbb{K}}]\right) \underline{\mathbf{u}}(\omega)=\underline{\mathbf{f}}(\omega)
$$

in which $\underline{\mathbf{u}}(\omega)$ and $\underline{\mathbf{f}}(\omega)$ are the $\mathbb{C}^{n}$ vectors of the DOF and of the external loads and where $[\underline{\mathbb{M}}],[\underline{\mathbb{D}}]$ and $[\underline{\mathbb{K}}]$ are the mean finite element mass, damping and stiffness matrices of the bladed-disk which are positive-definite matrices. The projection basis resulting from the substructuring method is written with the following block decomposition as

$$
\left[\begin{array}{l}
\underline{\mathbf{u}}_{i}^{d}(\omega) \\
\underline{\mathbf{u}}_{\Sigma}(\omega) \\
\underline{\mathbf{u}}_{i}^{b}(\omega)
\end{array}\right]=[\underline{H}]\left[\begin{array}{l}
\underline{\mathbf{q}}^{d}(\omega) \\
\underline{\mathbf{q}}^{b}(\omega)
\end{array}\right],[\underline{H}]=\left[\begin{array}{cc}
{\left[\underline{\Phi}_{i}^{d, c}\right]} & {[\mathbb{Q}]} \\
{\left[\underline{\Phi}_{\Sigma}^{d, c}\right]} & {[\mathbb{Q}]} \\
{\left[\underline{S}^{b}\right]\left[\underline{\Phi}_{\Sigma}^{d, c}\right]} & {\left[\underline{\Phi}^{b}\right]}
\end{array}\right]
$$

In Eq. (2), $\underline{\mathbf{u}}_{i}^{d}, \underline{\mathbf{u}}_{\Sigma}$ and $\underline{\mathbf{u}}_{i}^{b}$ are the $\mathbb{C}^{n_{i}^{d}}, \mathbb{C}^{N n_{\Sigma}}$ and $\mathbb{C}^{N n_{i}}$ vectors of the $n_{i}^{d}$ internal DOF of the disk, of the $N n_{\Sigma}$ coupling interface DOF and of the $N n_{i}$ internal DOF of the blades. The vectors $\underline{\mathbf{q}}^{d}$ and $\underline{\mathbf{q}}^{b}$ are the $\mathbb{C}^{N_{d}}$ and the $\mathbb{C}^{N_{b}}$ vectors of the generalized coordinates of the disk and of the blades. The block decompositions of matrices $\left[\underline{S}^{b}\right]$ and $\left[\underline{\Phi}^{b}\right]$ with respect to the blades are constituted of matrices $\left[\underline{S}_{j k}^{b}\right]=\left[\underline{S}^{j}\right] \delta_{j k}$ and $\left[\underline{\Phi}_{j k}^{b}\right]=\left[\underline{\Phi}^{j}\right] \delta_{j k}$ where subscript $j k$ is related to blade $j$ and blade $k$. Matrix $\left[\underline{\Phi}^{j}\right]$ is a matrix in $\mathbb{M}_{n_{i}, n_{b}}(\mathbb{R})$ whose columns are the eigenvectors related to the $n_{b}$ first eigenvalues of each blade with fixed coupling interface. The matrix $\left[\underline{S}^{j}\right]$ is a matrix in $\mathbb{M}_{n_{i}, n_{\Sigma}}(\mathbb{R})$ representing the static boundary functions. The matrices $\left[\underline{\Phi}_{i}^{d, c}\right]$ and $\left[\underline{\Phi}_{\Sigma}^{d, c}\right]$ correspond to the block decomposition of matrix $\left[\underline{\Phi}^{d, c}\right]$ with respect to the internal DOF and the coupling interface DOF of the disk. This matrix $\left[\underline{\Phi}^{d, c}\right]$ is the matrix in $\mathbb{M}_{N n_{\Sigma}+n_{i}^{d}, N_{d}}(\mathbb{R})$ whose columns are the eigenvectors of the disk loaded at its coupling interface with mass and stiffness terms issued from the static condensation of the blades. The integer $N_{d}$ is such that $N_{d}=N n_{d}$, in which $n_{d}$ is the number of loaded disk modes related to the $n_{d}$ first eigenvalues of the disk for a given circumferential wave number. It should be noted that such a load allows the disk modes to constitute a good approximation of the global modes of the structure. Such modes can be calculated by using the cyclic properties of the disk [4, 5]. Using (1) and (2) yields the mean reduced matrix equation

$$
\left[\underline{A}_{\mathrm{red}}(\omega)\right]\left[\underline{\mathbf{q}}^{b}(\omega)\right]=[\underline{H}]^{T} \underline{\mathbf{f}}(\omega)
$$

in which $\left[\underline{\mathbb{A}}_{\text {red }}(\omega)\right]=-\omega^{2}\left[\underline{\mathbb{M}}_{\text {red }}\right]+i \omega\left[\underline{\mathbb{D}}_{\text {red }}\right]+\left[\underline{\mathbb{K}}_{\text {red }}\right]$ is the mean reduced dynamic stiffness matrix of the bladed disk such that, for
$\mathbb{E}$ representing $\mathbb{M}, \mathbb{D}$ or $\mathbb{K}$,

$$
\left[\underline{\mathbb{E}}_{\mathrm{red}}\right]=[\underline{H}]^{T}[\underline{\mathbb{E}}][\underline{H}] \quad, \quad\left[\underline{\mathbb{E}}_{\mathrm{red}}\right] \in \mathbb{M}_{N_{b}+N_{d}}^{+}(\mathbb{R}) .
$$

Introducing the block decomposition associated with Eq. (3) yields

$$
\left[\underline{A}_{\mathrm{red}}(\omega)\right]=\left[\begin{array}{ll}
{\left[\underline{\mathcal{A}}^{d}(\omega)\right]} & {\left[\underline{A}_{c}(\omega)\right]} \\
{\left[\underline{A}_{c}(\omega)\right]^{T}} & {\left[\underline{\mathcal{A}}^{b}(\omega)\right]}
\end{array}\right],
$$

in which $\left[\underline{\mathcal{A}}^{d}(\omega)\right]$ is the generalized dynamic stiffness matrix of the disk constructed with eigenvectors matrix $\left[\underline{\Phi}^{d, c}\right]$ and where the block $j k$ of $\left[\underline{\mathcal{A}}^{b}(\omega)\right]$ is such that $\left[\underline{\mathcal{A}}^{b}(\omega)_{j k}\right]=\left[\underline{\mathcal{A}}^{j}(\omega)\right] \delta_{j k}$ in which $\left[\underline{\mathcal{A}}^{j}(\omega)\right]=-\omega^{2}\left[\underline{\mathcal{M}}^{j}\right]+i \omega\left[\underline{\mathcal{D}}^{j}\right]+\left[\underline{\mathcal{\mathcal { K }}}^{j}\right]$ defines the generalized dynamic stiffness matrix of each blade with fixed coupling interface and constructed with eigenvectors matrix $\left[\underline{\Phi}^{j}\right]$.

\section{MISTUNING ANALYSIS OF THE BLADED DISK WITH RANDOM UNCERTAINTIES}

A nonparametric probabilistic approach is used for modeling random uncertainties in each blade. Such a probabilistic approach consists in constructing a probability model on the reduced matrices of each blade. This probability model is derived from the maximization of the statistical entropy under constraints defined by the available information. The basis of this theory has been completely developed in linear elastodynamics for the lowfrequency range $[18,19]$ and for the medium-frequency range [23]. Furthermore, it has been extended and validated for modeling non homogeneous random uncertainties [24] and for modeling homogeneous statistical independent random uncertainties for blade mistuning problematics $[2,25]$.

\section{Nonparametric probabilistic model of random uncer- tainties}

Below, bold letters are related to deterministic vectors and bold capital letters are related to random vectors. Capital letters between brackets mean deterministic matrices and bold capital letters between brackets mean random matrices. Since the blade is reduced by using the Craig and Bampton method, it can be shown that the reduced matrix equation of tuned blade $j$ with free coupling interface is written as

$$
\left[\begin{array}{cc}
{\left[\underline{\mathcal{A}}^{j}(\omega)\right]} & {\left[\underline{A}_{S}^{j}(\omega)\right]} \\
{\left[\underline{A}_{S}^{j}(\omega)\right]^{T}} & {\left[\underline{A}_{\Sigma}^{j}(\omega)\right]}
\end{array}\right]\left[\begin{array}{l}
\underline{\mathbf{q}}^{j}(\omega) \\
\underline{\mathbf{u}}_{\Sigma}^{j}(\omega)
\end{array}\right]=\underline{\mathcal{F}}^{j}(\omega)
$$

in which for blade $j, \underline{\mathbf{u}}_{\Sigma}^{j}$ and $\underline{\mathbf{q}}^{j}$ are the vectors of the coupling interface DOF and of the generalized coordinates. Matrix block $\left[\underline{\mathcal{A}}^{j}(\omega)\right]$ corresponds to the dynamic part of the mean reduced matrix model for blade $j$ with fixed coupling interface. For 
each blade, it is assumed that random uncertainties mainly affect the dynamics of the blade with fixed coupling interface [1]. Consequently, the nonparametric probabilistic approach is implemented with respect to the matrix $\left[\underline{\mathcal{A}}^{j}(\omega)\right]$. The nonparametric model of random uncertainties is then written as

$$
\mathbf{U}(\omega)=[\underline{H}]\left[\begin{array}{l}
\mathbf{Q}^{d}(\omega) \\
\mathbf{Q}^{b}(\omega)
\end{array}\right],
$$

in which $\mathbf{Q}^{d}$ is the $\mathbb{C}^{N_{d}}$-valued vector of the random generalized coordinates of the disk and where $\mathbf{Q}^{b}=\left(\mathbf{Q}^{0}, \ldots, \mathbf{Q}^{N-1}\right)$ is the $\mathbb{C}^{N_{b}}$-valued vector of the random generalized coordinates of the blades. Random vector $\left(\mathbf{Q}^{d}, \mathbf{Q}^{b}\right)$ is the solution of the random reduced matrix equation

$$
\left[\begin{array}{ll}
{\left[\underline{\mathcal{A}}^{d}(\omega)\right]} & {\left[\underline{A}_{c}(\omega)\right]} \\
{\left[\underline{A}_{c}(\omega)\right]^{T}} & {\left[\mathcal{A}^{b}(\omega)\right]}
\end{array}\right]\left[\begin{array}{l}
\mathbf{Q}^{d}(\omega) \\
\mathbf{Q}^{b}(\omega)
\end{array}\right]=\left[\underline{H}^{T} \underline{\mathbf{f}}(\omega),\right.
$$

in which the block $j k$ of $\left[\mathcal{A}^{b}(\omega)\right]$ is such that $\left[\mathcal{A}^{b}(\omega)_{j k}\right]=$ $\left[\mathcal{A}^{j}(\omega)\right] \delta_{j k}$ and where $\left[\mathcal{A}^{j}(\omega)\right]$ is the random reduced dynamic stiffness matrix of blade $j$ defined by $\left[\mathcal{A}^{j}(\omega)\right]=-\omega^{2}\left[\mathcal{M}^{j}\right]+$ $i \omega\left[\mathcal{D}^{j}\right]+\left[\mathcal{K}^{j}\right]$. The available information for random matrices $\left[\mathcal{M}^{j}\right],\left[\mathcal{D}^{j}\right]$ and $\left[\mathcal{K}^{j}\right]$ is

$$
\begin{array}{r}
\mathcal{E}\left\{\left[\mathcal{M}^{j}\right]\right\}=\left[\underline{\mathcal{M}}^{j}\right], \mathcal{E}\left\{\left[\mathcal{D}^{j}\right]\right\}=\left[\underline{\mathcal{D}}^{j}\right], \mathcal{E}\left\{\left[\mathcal{K}^{j}\right]\right\}=\left[\underline{\mathcal{K}}^{j}\right] \\
{\left[\mathcal{M}^{j}\right],\left[\mathcal{D}^{j}\right],\left[\mathcal{K}^{j}\right] \text { are } \mathbb{M}_{n_{a}}^{+}(\mathbb{R}) \text {-valued random matrices }} \\
\mathcal{E}\left\{\left\|\left[\mathcal{M}^{j}\right]^{-1}\right\|_{F}^{2}\right\}<+\infty, \mathcal{E}\left\{\left\|\left[\mathcal{D}^{j}\right]^{-1}\right\|_{F}^{2}\right\}<+\infty \\
\mathcal{E}\left\{\left\|\left[\mathcal{K}^{j}\right]^{-1}\right\|_{F}^{2}\right\}<+\infty
\end{array}
$$

in which $\mathcal{E}$ is the mathematical expectation and where $\|[A]\| \|_{F}$ denotes the Frobenius norm of matrix $[A]$. The use of the maximum entropy principle with the constraints defined by this available information allows the probability distribution of each random matrix to be constructed and it can be proved that $\left[\mathcal{M}^{j}\right],\left[\mathcal{D}^{j}\right]$, $\left[\mathcal{K}^{j}\right], j \in\{0, \ldots, N-1\}$ are independent random variables. It should be noted that these constraints allow the random response $\mathbf{U}(\omega)$ to be a second-order random vector. All the details concerning the construction of this probability model can be found in $[18,19]$.

\section{Identification of the dispersion parameters of the non- parametric approach with respect to the tolerance}

For each blade $j$, the probability distribution of each random matrix depends only on dimension $n_{a}$ and on a positive parameter $\delta^{j}$ called the dispersion parameter. Consequently, for a given blade $j$, there are three positive numbers $\delta_{M}^{j}, \delta_{D}^{j}$ and $\delta_{K}^{j}$ which are the dispersion parameters controlling the dispersion level of the three independent random matrices $\left[\mathcal{M}^{j}\right],\left[\mathcal{D}^{j}\right]$ and $\left[\mathcal{K}^{j}\right]$. The use of these dispersion parameters as parameters of the probability distribution of the random matrices is explained in [19]. It should be noted that the probability distribution of each random matrix is a strongly nonlinear function of its dispersion parameter. Since we are interested in studying the effects of mistuning due to blade manufacturing tolerances, the dispersion parameters have to be quantified with respect to the tolerances specifications. Such an identification is achieved in constructing a random geometry of the blade which respects the tolerances specifications. The random geometry model allows the random mass matrix $\left[\mathbf{M}^{\text {para, } j}\right]$ and the random stiffness matrix $\left[\mathbf{K}^{\text {para, } j}\right]$ to be constructed for blade $j$. It can be shown [1] that dispersion parameters $\delta_{M}^{j}$ and $\delta_{K}^{j}$ can be identified by the equation

$$
\begin{aligned}
& \delta_{M}^{j}=\sqrt{\frac{W_{M}^{\mathrm{tol}, j}\left(n_{b}+1\right)}{\operatorname{tr}\left(\left[\underline{\mathcal{M}}^{j}\right]^{2}\right)+\operatorname{tr}\left(\left[\underline{\mathcal{M}}^{j}\right]\right)^{2}}}, \\
& \delta_{K}^{j}=\sqrt{\frac{W_{K}^{\mathrm{tol}, j}\left(n_{b}+1\right)}{\operatorname{tr}\left(\left[\underline{\mathcal{K}}^{j}\right]^{2}\right)+\operatorname{tr}\left(\left[\underline{\mathcal{K}}^{j}\right]\right)^{2}}},
\end{aligned}
$$

in which tr denotes the trace and where $W_{M}^{\mathrm{tol}, j}$ and $W_{K}^{\mathrm{tol}, j}$ are defined by

$$
\begin{aligned}
W_{M}^{\mathrm{tol}, j} & =\mathcal{E}\left\{\left\|\left[\underline{\Phi}^{j}\right]^{T}\left[\mathbf{M}^{\mathrm{para}, j}\right]\left[\underline{\Phi}^{j}\right]-\left[\underline{\mathcal{M}}^{j}\right]\right\|_{F}^{2}\right\} \\
W_{K}^{\mathrm{tol}, j} & =\mathcal{E}\left\{\left\|\left[\underline{\Phi}^{j}\right]^{T}\left[\mathbf{K}^{\mathrm{para}, j}\right]\left[\underline{\Phi}^{j}\right]-\left[\underline{\mathcal{K}}^{j}\right]\right\|_{F}^{2}\right\}
\end{aligned}
$$

It should be noted that Eqs. (12) and (14) (or Eqs. (13) and (15)) do not assume that the mean value of random mass matrix $\left[\mathbf{M}^{\text {para, } j}\right]$ (or random stiffness matrix $\left[\mathbf{K}^{\text {para, } j}\right]$ ) is equal to the corresponding matrix of the nominal model. Consequently, the calculation of the dispersion parameters includes the bias between the mean value and the nominal value. This assumption corresponds to the reality: there are no reasons for which the mean value over a set of several blades manufactured with a given specification of tolerances coincides with the nominal blade used for the mean model and used for the manufacturing (mainly because the stiffness and the mass matrix do not linearly depend on the geometry). The Monte-Carlo numerical simulation is used for computing $\delta_{M}^{j}$ and $\delta_{K}^{j}$. Once the dispersion parameters are identified, the stochastic equation defined by Eq. (8) is solved with the Monte-Carlo numerical simulation.

\section{Random dynamic magnification factor}

Let $\left[\underline{K}^{j}\right]$ be the mean finite element stiffness matrix of blade $j$. The elastic energy of blade $j$ related to the mean dynamical system is written as $\underline{e}^{j}(\omega)=\frac{1}{2} \underline{\mathbf{u}}^{j}(\omega)^{*}\left[\underline{K}^{j}\right] \underline{\mathbf{u}}^{j}(\omega)$, in which $\underline{\mathbf{u}}^{j}$ is the vector of the DOF related to tuned blade $j$. Due to the cyclic symmetry, we have $\underline{e}^{0}(\omega)=\ldots=\underline{e}^{N-1}(\omega)$ denoted as $\underline{e}(\omega)$. The similar quantity related to the stochastic dynamic system is defined as $\mathbf{E}^{j}(\omega)=\frac{1}{2} \mathbf{U}^{j}(\omega)^{*}\left[\underline{K}^{j}\right] \mathbf{U}^{j}(\omega)$, in which $\mathbf{U}^{j}$ is the random vector of the DOF related to mistuned blade $j$. For $\omega$ fixed in $\mathbb{B}$, the random dynamic analysis is carried out by introducing the random dynamic magnification factor $\mathbf{B}(\omega)$ defined 
with respect to the elastic energy by

$$
\mathbf{B}(\omega)=\max _{j=0, \ldots, N-1} \sqrt{\frac{\mathbf{E}^{j}(\omega)}{\underline{e}_{\infty}}} \quad, \quad \underline{e}_{\infty}=\max _{\omega \in \mathbb{B}} \underline{e}(\omega)
$$

Moreover, the random dynamic magnification factor over frequency band $\mathbb{B}$ is defined by

$$
B_{\infty}=\max _{\omega \in \mathbb{B}} \mathbf{B}(\omega)
$$

We are interested in estimating the probability density function $b \mapsto p_{B_{\infty}}(b)$ and the probability $\mathcal{P}\left(B_{\infty}>b_{\infty}\right)$, in which $b_{\infty}$ is a given amplification level. A convergence analysis with respect to parameters $n_{d}$ and $n_{b}$ allowing the reduction of the model to be controlled is then carried out. The stochastic convergence analysis is limited to the second-order convergence of $B_{\infty}$ because it implies the convergence in law. We then introduce the mapping $\left(n_{d}, n_{b}\right) \mapsto||\left|B_{\infty}\right|||$ with $\|\left|B_{\infty}\right|||^{2}=\mathcal{E}\left\{B_{\infty}^{2}\right\}$. This function $\left\|\left|B_{\infty} \|\right|\right.$ is estimated by $\operatorname{Conv}\left(n_{s}, n_{d}, n_{b}\right)$ such that

$$
\operatorname{Conv}^{2}\left(n_{s}, n_{d}, n_{b}\right)=\frac{1}{n_{s}} \sum_{i=1}^{n_{s}} B_{\infty}^{2}\left(\theta_{i}\right)
$$

in which $\theta_{1}, \ldots, \theta_{n_{s}}$ denote the $n_{s}$ realizations of the Monte-Carlo numerical simulation.

\section{APPLICATION TO AN INDUSTRIAL FAN STAGE Description of the structure}

The structure under consideration is a wide chord supersonic fan geometry called SGC1. The fan has 22 blades. The finite element model of the bladed disk is shown in Fig. 1.

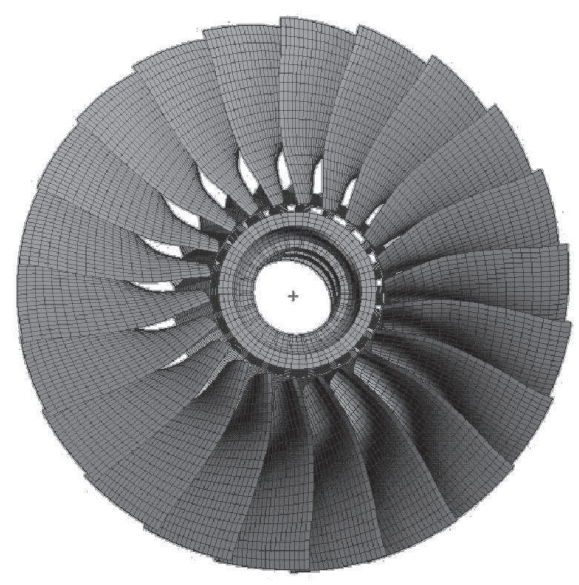

Figure 1. Finite element mesh for the 22 blades fan stage.
It is constructed with 31812 solid elements and is constituted of $n=473814$ DOF. Each sector contains 8133 nodes which corresponds to 22947 DOF. The structure is also characterized by the following parameters $n_{i}=16197, n_{\Sigma}=414$ and $n_{i}^{d}=6036$. The structure is in rotation around its revolution axis with a constant velocity $\Omega=4500 \mathrm{rpm}$. Since the dynamic analysis is carried out in the rotating frame of the structure, the rigidbody motion due to the rotation of the structure corresponds to a fixed boundary condition at the inner radius of the structure. The bladed disk is made of titanium. The finite element matrices which include the prestresses due to the rotational motion are generated for one sector. The cyclic symmetry is used in order to construct the mean reduced matrices described in Eq. (5) and allows the generalized eigenvalue problem related to the tuned bladed disk to be solved. Figure 2 displays the eigenfrequencies of the tuned bladed disk with respect to the circumferential wave number. In this work, we are interested in studying the mistuned forced response in a low-frequency band of analysis defined by $\mathbb{B}=[515,545] \mathrm{Hz}$ with a third engine order excitation. A damping model is added to the bladed disk which corresponds to a hysteretic model with a mean loss factor $\eta=0.002$.

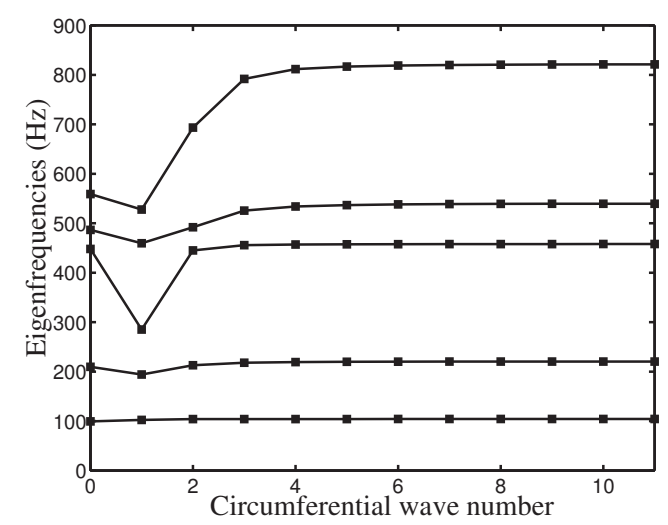

Figure 2. Graph of the eigenfrequencies with respect to the circumferential wave number for the tuned structure

\section{Stochastic convergence analysis for the random re- duced model}

For $\delta_{M}^{j}=\delta_{D}^{j}=0$ and $\delta_{K}^{j}=0.05$, Figs 3 and 4 are relative to the convergence analysis of $\|\left|B_{\infty}\right|||$ with respect to parameters $n_{d}$ and $n_{a}$ allowing the dimension of the random reduced matrix model to be controlled and with respect to the number $n_{s}$ of realizations of the Monte-Carlo numerical simulation. Figure 3 displays the graph $n_{s} \mapsto \operatorname{Conv}^{2}\left(n_{s}, n_{d}, n_{b}\right)$ for $n_{d}=10$ and $n_{b}=20$. A reasonable convergence is obtained for $n_{s}=300$. For $n_{s}=300$, Figure 4 shows the graph $n_{b} \mapsto \operatorname{Conv}^{2}\left(300, n_{d}, n_{b}\right)$ for $n_{d}=10$ (black solid line), for $n_{d}=7$ (gray solid line), for $n_{d}=5$ (black dashed-dotted line), for $n_{d}=4$ (gray dashed-dotted line), for $n_{d}=3$ (black dotted line) and for $n_{d}=2$ (gray dotted line). It 
can be deduced that $n_{d}=7$ and $n_{b}=7$ constitute the optimal values of the $n_{d}$ and of the $n_{b}$ parameters for the mistuning analysis of this industrial fan. It should be noted that such a reduction allows the dimension of the stochastic finite element model to be reduced of a factor around 1500 .

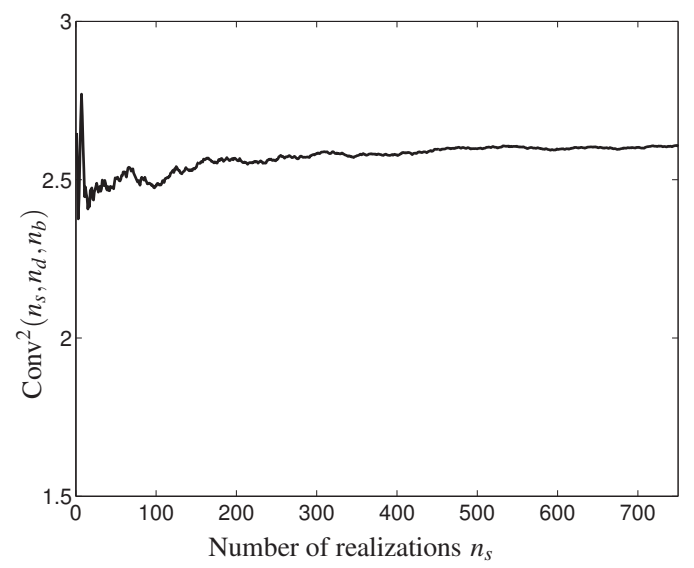

Figure 3. Graph of function $n_{s} \mapsto \operatorname{Conv}^{2}\left(n_{s}, n_{d}, n_{b}\right)$ related to the stochastic dynamic equation with $\delta_{M}^{j}=\delta_{D}^{j}=0$ and $\delta_{K}^{j}=0.05$ and for $n_{d}=10$ and $n_{b}=20$.

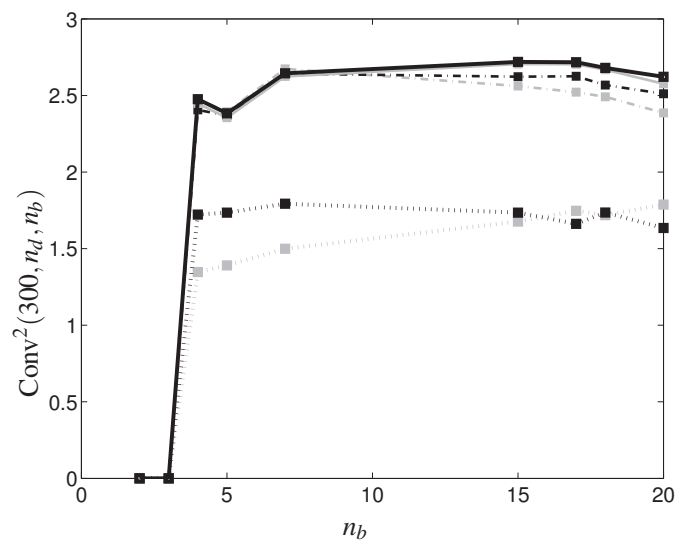

Figure 4. Graph of function $n_{b} \mapsto \operatorname{Conv}^{2}\left(300, n_{d}, n_{b}\right)$ for $n_{d}=10$ (black solid line), for $n_{d}=7$ (gray solid line), for $n_{d}=5$ (black dashed-dotted line), for $n_{d}=4$ (gray dashed-dotted line), for $n_{d}=3$ (black dotted line) and for $n_{d}=2$ (gray dotted line). and related to the stochastic dynamic equation with $\delta_{M}^{j}=\delta_{D}^{j}=0$ and $\delta_{K}^{j}=0.05$

\section{Identification of the dispersion parameters}

It results from the manufacturing process of a blade that all manufactured blades are different from one to another one and from the nominal blade. The tolerances which characterize the authorized fluctuations of the blade geometry are defined from its nominal geometry. In the context of the blade manufacturing, the tolerances are specified for different sections at a given height from the base of the blade. Let $S_{1}, S_{2}$ and $S_{3}$ be three section profiles defined in Fig. 5. For $k=1,2,3$, the parameters considered in this paper are the length $L_{k}$ of the chord and an angular torsion $\alpha_{k}$ which allows the shape of the trailing edge to be controlled (see Figure 6). A random blade geometry has to be constructed

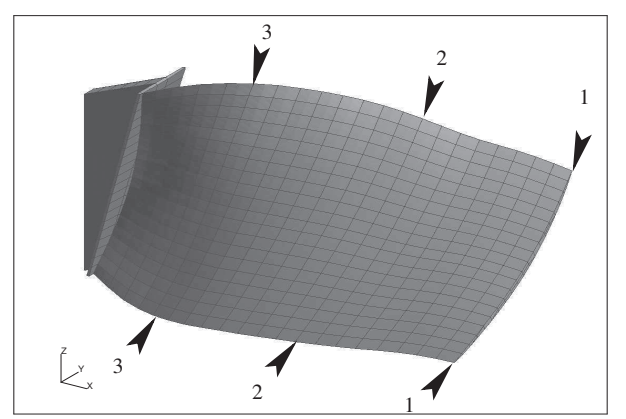

Figure 5. Mesh of the nominal blade and definition of three section profiles

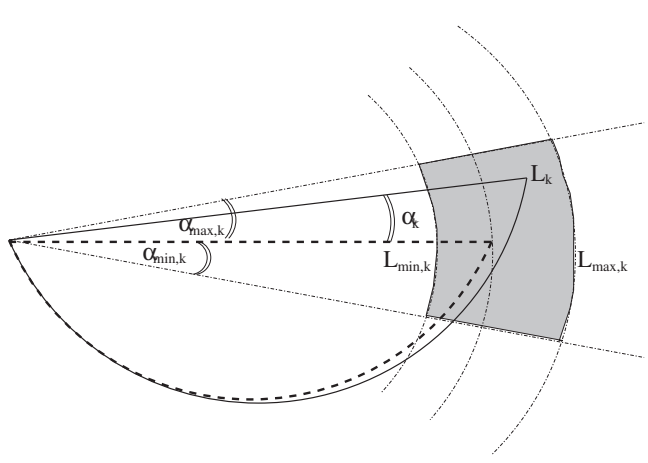

Figure 6. Geometrical parameters of the tolerances: section $S_{k}$ of the nominal blade (thick dashed-line) and of the manufactured blade (thick solid line). Location of the trailing edge (gray filled zone) with respect to the tolerances specifications.

in order to identify the dispersion parameters of the nonparametric model of random uncertainties. Such a random geometry has to be representative of a manufactured blade. It means that the random geometry has to respect the constraints due to tolerances specifications and has to have a certain regularity of shape from one section to another one. Let $\underline{\mathbf{x}}$ be the vector in $\mathbb{R}^{3\left(n_{i}+n_{\Sigma}\right)}$ related to the coordinates of the nodes belonging to the nominal 
mesh of the blade. Let then $\mathbf{x}$ be the vector in $\mathbb{R}^{3\left(n_{i}+n_{\Sigma}\right)}$ related to the coordinates of the nodes belonging to the mesh of the manufactured blade. In the probabilistic context of tolerancing, we then model $\mathbf{x}$ by the $\mathbb{R}^{3\left(n_{i}+n_{\Sigma}\right)}$-valued random variable $\mathbf{X}$. The random fluctuation of the geometry is generated by the following model

$$
\mathbf{X}-\underline{\mathbf{x}}=\sum_{i \in \mathcal{I}} T_{i} \varphi_{i}
$$

in which $\left\{\boldsymbol{\varphi}_{i}, i \in \mathcal{I}\right\}$ is a vector basis chosen as a subset $\mathcal{I}$ of the set of the eigenmodes of the nominal blade with fixed coupling interface and where $T_{i}, i \in \mathcal{I}$ are independent random variables with a uniform distribution. Figure 7 shows several section profiles for one realization of the random blade geometry with an amplification coefficient equal to 20 (gray filled thin line) and for the nominal blade (thick line). It should be noted that the construction of such a probabilistic model of the geometry remains arbitrary and does not constitute a model corresponding to a given manufacturing process. Nevertheless, this model is not directly used to carry out a direct mistuning analysis. It is important to note that the random dynamic magnification factor is strongly sensitive to the choice of the probability model which is constructed with the nonparametric approach and consequently does not directly depend on the probabilistic model of the geometry: this nonparametric model depends only on the dispersion parameters. The probabilistic model of the geometry allows these dispersion parameters to be constructed as a function of the tolerances. An error on this function induces an error on the value of the dispersion parameters for a given tolerance but does not yield an error on the mistuning analysis for a given value of the dispersion parameters.

Let $p \in\left\{1, \ldots, n_{i}+n_{\Sigma}\right\}$ be a node of the mesh of the blade. The block decomposition of vectors $\underline{\mathbf{x}}$ and $\mathbf{x}$ with respect to a node $p$ of the mesh is denoted as $\underline{\mathbf{x}}_{p}$ and $\mathbf{x}_{p}$ which are vectors in $\mathbb{R}^{3}$. Let $n_{k}$ be the node related to the trailing edge of $S_{k}, k \in\{1,2,3\}$. The tolerance specifications allows parameter $L_{k}$ (or parameter $\alpha_{k}$ ) to be bounded between $L_{\min , k}$ and $L_{\max , k}$ (or between $\alpha_{\min , k}$ and $\alpha_{\max , k}$ ). The coordinates of node $n_{k}$ are then bounded such that

$$
\left|\mathbf{x}_{n_{k}}-\underline{\mathbf{x}}_{n_{k}}\right| \leq \Delta \underline{\mathbf{c}}_{k}, \forall k \in\{1,2,3\} \quad,
$$

in which $\Delta \underline{\mathbf{c}}_{k}$ is the vector in $\mathbb{R}^{3}$ related to the bounds of $\mathbf{x}_{n_{k}}$ induced by the tolerance specifications characterized by $L_{\min , k}$, $\alpha_{\min , k}, L_{\max , k}$ and $\alpha_{\max , k}$. The random mesh of the blade is modeled by assuming that all the nodes of the mesh are allowed to fluctuate with respect to the nominal mesh. The probability distributions $p_{T_{i}}\left(t_{i}\right)$ of uniform random variables $T_{i}, i \in \mathcal{I}$ are then estimated by the Monte-Carlo numerical simulation with an optimization procedure. Let $\theta_{1}, \ldots, \theta_{n_{s}}$ be the $n_{s}$ realizations used by the Monte-Carlo numerical simulation. We have then to find $\forall i \in \mathcal{I}$, the probability distribution $p_{T_{i}}\left(t_{i}\right)$ of uniform random variable $T_{i}$ such that $\forall p \in\left\{1, \ldots, n_{i}+n_{\Sigma}\right\}$ and $\forall j \in\left\{1, \ldots, n_{s}\right\}$

$$
\left|\sum_{i \in \mathcal{I}} T_{i}\left(\theta_{j}\right) \boldsymbol{\varphi}_{i, p}\right| \leq \min _{k \in\{1,2,3\}}\left(\Delta \mathbf{c}_{k}\right)
$$

in which $\varphi_{i, p}$ is the restriction of vector $\varphi_{i}$ on the DOF of node $p$ and where $T_{i}\left(\theta_{j}\right)$ is the realization number $j$ of random variable $T_{i}$. It follows that this random geometry model is in agreement with the tolerance specifications described in Eq. (20).

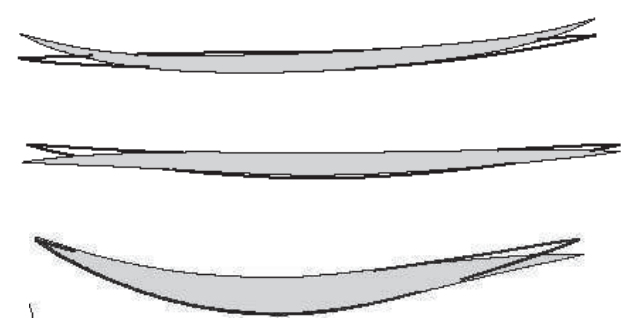

Figure 7. Section profiles for a realization of the manufactured blade with a zoom $20 \times$ (gray filled thin line) and for the nominal blade (thick line)

The implementation of this strategy is carried out by using the Monte-Carlo numerical simulation with an heterodyne strategy for computing the random variables in order to accelerate the stochastic convergence.

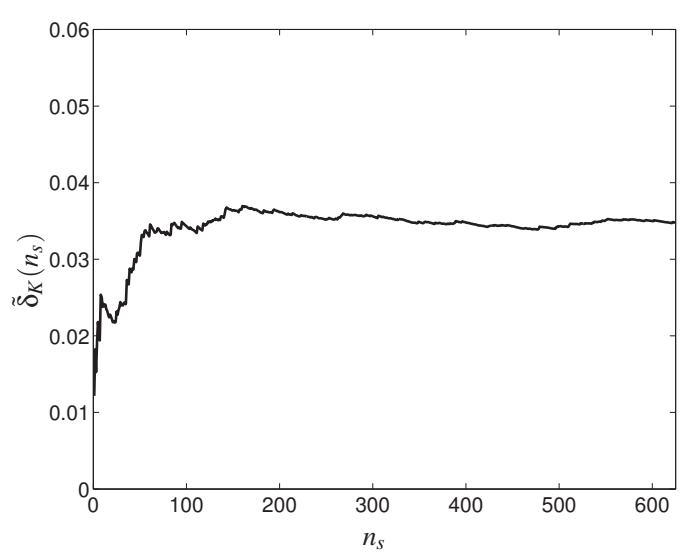

Figure 8. Convergence with respect to the number $n_{s}$ of realizations: Graph of function $n_{s} \mapsto \tilde{\delta}_{K}\left(n_{s}\right)$ for $d L_{\min }=-0.55 \mathrm{~mm}, d L_{\max }=0.75 \mathrm{~mm}, \alpha_{\max }=$ $-\alpha_{\min }=0.55$ degrees.

Let us introduce $d L_{\min , k}$ and $d L_{\max , k}$ such that $d L_{\min , k}=$ $L_{k, 0}-L_{\min , k}$ and $d L_{\max , k}=L_{\max , k}-L_{k, 0}$ in which, for section $S_{k}$, the parameter $L_{k, 0}$ is the length of the chord related to the nominal blade. In the numerical application, we have $d L_{\min , 1}=$ 
$\ldots=d L_{\min , 3}$ denoted as $d L_{\min }, d L_{\max , 1}=\ldots=d L_{\max , 3}$ denoted as $d L_{\max }, \alpha_{\min , 1}=\ldots=\alpha_{\min , 3}$ denoted as $\alpha_{\min }$ and $\alpha_{\max , 1}=\ldots=\alpha_{\max , 3}$ denoted as $\alpha_{\max }$. We introduce then Figure 8 and 9 are related to the convergence with respect to the number $n_{s}$ of realizations and display the graphs $n_{s} \mapsto \tilde{\delta}_{K}\left(n_{s}\right)$ and $n_{s} \mapsto \tilde{\delta}_{M}\left(n_{s}\right)$ in which $\tilde{\delta}_{K}\left(n_{s}\right)$ and $\tilde{\delta}_{M}\left(n_{s}\right)$ are the statistical estimators of Eq. $(12,13)$ for $d L_{\min }=-0.55 \mathrm{~mm}, d L_{\max }=0.75 \mathrm{~mm}$, $\alpha_{\max }=-\alpha_{\min }=0.55$ degrees. A reasonable convergence is obtained for $n_{s}=250$. It is then deduced that the dispersion parameters corresponding to the values of the tolerances parameters are such that $\delta_{K}=0.035$ and $\delta_{M}=3 \times 10^{-5}$.

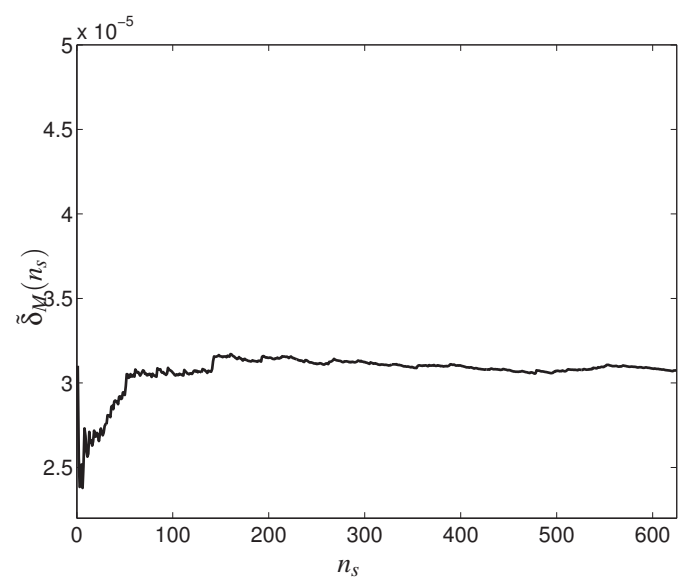

Figure 9. Convergence with respect to the number $n_{s}$ of realizations: Graph of function $n_{s} \mapsto \tilde{\delta}_{M}\left(n_{s}\right)$ for $d L_{\min }=-0.55 \mathrm{~mm}, d L_{\max }=0.75 \mathrm{~mm}, \alpha_{\max }=$ $-\alpha_{\min }=0.55$ degrees.

\section{Probabilistic analysis of the blade mistuning due to ge- ometric uncertainties.}

In this Section, the random mistuning analysis is carried out for the complete bladed disk. In this application, it is assumed that the random uncertainties are homogeneous from one blade to another one yielding $\delta_{M}^{j}=\delta_{M}, \delta_{D}^{j}=\delta_{D}$ and $\delta_{K}^{j}=\delta_{K}$, for all $j \in$ $\{0, \ldots, N-1\}$. The mass and the stiffness dispersion parameters induced by the random geometry of the blade are then deduced from the identification procedure above. Such an identification methodology does not include damping uncertainty. Therein, it will be assumed that the damping dispersion parameter $\delta_{D}$ is 0 . The Monte-Carlo numerical simulation is performed with $n_{s}=$ 1500 realizations.

Figure 10 shows the probability density function $b \mapsto$ $p_{B_{\infty}}(b)$ in the case of the blade mistuning is only due to the variation of the chord length related to each blade profile. With $d L_{\min }=-0.55 \mathrm{~mm}$ and $d L_{\max }=0.75 \mathrm{~mm}$ and $\alpha_{\max }=\alpha_{\min }=0$, it is found that the corresponding dispersion parameters are very close to 0 and do not yield significant amplification with respect to the tuned case.

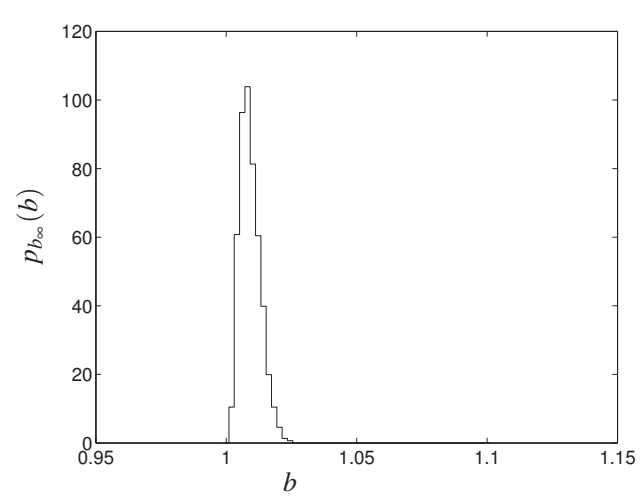

Figure 10. Graph of the probability density function $b \mapsto p_{B_{\infty}}(b)$ for $d L_{\min }=$ $-0.55 \mathrm{~mm}$ and $d L_{\max }=0.75 \mathrm{~mm}, \alpha_{\max }=-\alpha_{\min }=0$ degree.

A sensitivity analysis with respect to the tolerances related to the blade torsion angle is then considered. The level of uncertainty related to the blade chord uncertainty is given by $d L_{\min }=-0.55 \mathrm{~mm}$ and $d L_{\max }=0.75 \mathrm{~mm}$. Figure 11 and figure 12 display the probability density function $b \mapsto p_{B_{\infty}}(b)$ for $\alpha_{\max }=-\alpha_{\min }=0.35$ and $\alpha_{\max }=-\alpha_{\min }=0.55$ which correspond with the following dispersion parameters $\delta_{M}=2 \times 10^{-5}$, $\delta_{K}=0.015$ and $\delta_{M}=3 \times 10^{-5}, \delta_{K}=0.035$ respectively.

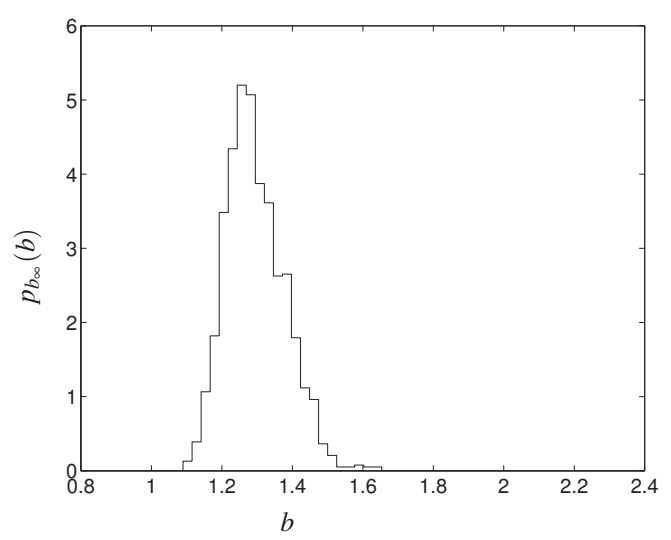

Figure 11. Graph of the probability density function $b \mapsto p_{B_{\infty}}(b)$ for $d L_{\min }=$ $-0.55 \mathrm{~mm}$ and $d L_{\max }=0.75 \mathrm{~mm}, \alpha_{\max }=-\alpha_{\min }=0.35$ degree.

It shows that there exist configurations of the random geometry which yield amplification factors upper than 1.6. Furthermore, the probability distribution of the amplification factor is very sensitive with respect to $\alpha_{\max }$ which allows the variability related to the twist of the blade to be controlled. It should be noted that due to random tolerances, the amount of uncertainty is mainly distributed on the stiffness because the value of the mass dispersion parameter is less than 1000 times the stiffness disper- 
sion parameter.

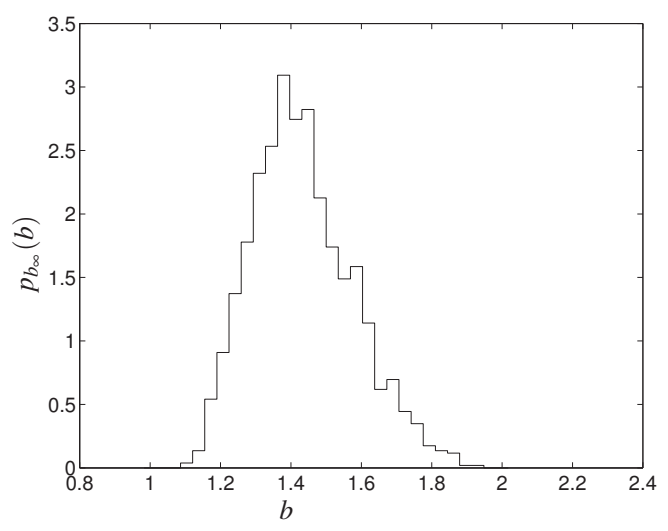

Figure 12. Graph of the probability density function $b \mapsto p_{B_{\infty}}(b)$ for $d L_{\min }=$ $-0.55 \mathrm{~mm}$ and $d L_{\max }=0.75 \mathrm{~mm}, \alpha_{\max }=-\alpha_{\min }=0.55$ degree.

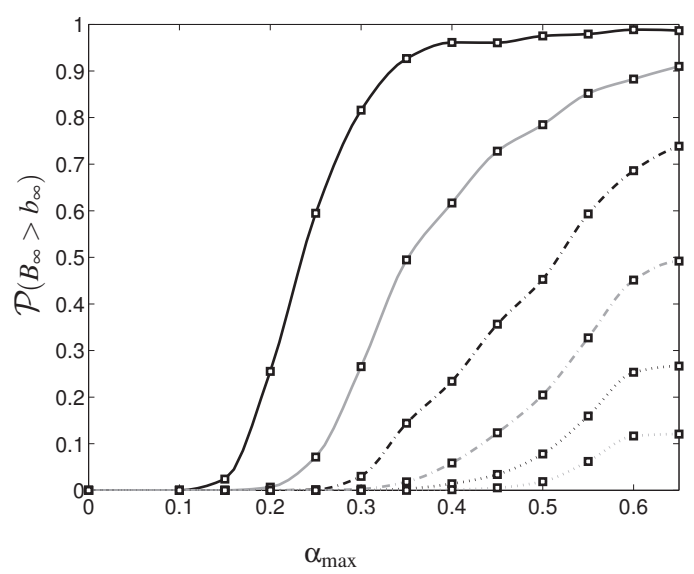

Figure 13. Graph of $\alpha_{\max } \mapsto \mathcal{P}\left(B_{\infty}>b_{\infty}\right)$ for several values of $b_{\infty}=1.2$ (black solid line), $b_{\infty}=1.3$ (gray solid line), $b_{\infty}=1.4$ (black dashed-dotted line), $b_{\infty}=1.5$ (gray dashed-dotted line), $b_{\infty}=1.6$ (black dotted line) and $b_{\infty}=1.7$ (gray dotted line).

Figure 13 displays the graphs $\alpha_{\max } \mapsto \mathcal{P}\left(B_{\infty}>b_{\infty}\right)$ for several values of $b_{\infty}=1.2$ (black solid line), $b_{\infty}=1.3$ (gray solid line), $b_{\infty}=1.4$ (black dashed-dotted line), $b_{\infty}=1.5$ (gray dashed-dotted line), $b_{\infty}=1.6$ (black dotted line) and $b_{\infty}=1.7$ (gray dotted line). It was seen on a simple numerical example [1] that this kind of graph has a maximum which allows two types of specifications to be considered. One of this specification deals with defining tolerances with high precision, the other one is related to intentional detuning of the blade. Nevertheless, in this industrial application, only the monotonic part of the graph is investigated. From this graph, it can be read for $\alpha_{\max }=0.55$ degree that we have $\mathcal{P}\left(B_{\infty}>1.6\right)=0.1593, \mathcal{P}\left(B_{\infty}>1.5\right)=$ 0.3273 and $\mathcal{P}\left(B_{\infty}>1.4\right)=0.5933$. Furthermore, it can be seen that the confidence region defined by $\mathcal{P}\left(B_{\infty}>1.5\right) \leq 0.1$ is obtained for parameter $\alpha_{\max }<0.43$ degree.

\section{CONCLUSIONS}

This paper deals with the specification of the blade geometrical tolerances for mistuned bladed disks by using a nonparametric probabilistic approach. The theory which has been previously developed for a simple numerical example is applied to an industrial bladed disk. First, a mean reduced model of the bladed disk is constructed with a "branch mode" substructuring method. This model is adapted for implementing the nonparametric methodology for each uncertain substructure. Mistuning is then introduced by using a nonparametric probabilistic approach. It should be noted that such a probabilistic model allows the random matrices to be positive definite which is coherent with the linear structural dynamic theory. Furthermore, the nonparametric approach allows both blade eigenfrequencies and blade eigenvectors to be represented with coherence. For each blade, the quantification of these uncertainties is described by three scalar parameters which allow the global dispersion of mass, damping and stiffness random matrices to be controlled. In this paper, we are interested by solving the inverse problem which consists in characterizing the geometrical tolerances for a given confidence region of the random dynamic magnification factor over a narrow-frequency band of analysis. Such a method requires to relate the dispersion parameters of the nonparametric probabilistic approach to the tolerance parameters of the blades. It should be noted that if the random dynamic magnification factor of the mistuned bladed disk is strongly sensitive to the nonparametric probability model of random uncertainties, then it is weakly sensitive to the probability model for the random geometry which is only used to estimate the dispersion parameters of the nonparametric model as a function of the tolerance parameters. This is the reason why an arbitrary coherent probability model of the blade random geometry can be chosen. Once the dispersion parameters are quantified, the probability levels concerning the random dynamic magnification factor are then characterized with respect to the specified tolerances. This paper proposes a methodology allowing the dynamic amplification induced by mistuning to be fixed. Finally, the proposed method based on the use of the nonparametric approach could be extended to the case of correlated random uncertainties between blades.

\section{REFERENCES}

[1] Capiez-Lernout, E., and Soize, C., 2003. "Specifying manufacturing tolerances for a given amplification factor: a nonparametric probabilistic methodology". In ASME Paper GT-2003-38050, Proceedings of ASME Turbo Expo Land Sea and Air, Atlanta, Georgia. 
[2] Capiez-Lernout, E., and Soize, C., 2002. "Nonparametric modeling of random uncertainties for dynamic response of structures with cyclic geometry. application to mistuned bladed-disks.". ASME Journal of Engineering for Gas Turbines and Power, submitted in June 2002, revised in September 2003.

[3] Seinturier, E., Lombard, J.-P., Berthillier, M., and Sgarzi, O., 2002. "Turbine mistuned forced response prediction comparison with experimental results". In ASME paper GT-2002-30424, Proceedings of ASME Turbo Expo Land Sea and Air, Amsterdam, the Netherlands.

[4] Thomas, D., 1979. "Dynamics of rotationally periodic structures". International Journal for Numerical Methods in Engineering, 14, pp. 81-102.

[5] Ohayon, R., and Soize, C., 1998. Structural acoustics and vibration. Academic press.

[6] Whitehead, D., 1966. "Effects of mistuning on the vibration of turbomachine blades induced by wakes". Journal of Mechanical Engineering Science, 8 (1) January, pp. 15-21.

[7] Ewins, D., 1969. "The effects of detuning upon the forced vibrations of bladed disks". JSV, 9 (1) January , pp. 65-69.

[8] Dye, R., and Henry, T., 1969. "Vibration amplitudes of compressor blades resulting from scatter in blade natural frequencies". ASME Journal of Engineering for Power, 91 (3) July , pp. 182-187.

[9] Lin, C., and Mignolet, M., 1997. "An adaptative perturbation scheme for the analysis of mistuned bladed disks". ASME Journal of Engineering for Gas Turbines and Power, 119 (1), pp. 153-160.

[10] Mignolet, M., Lin, C., and LaBorde, B., 2001. “A novel limit distribution for the analysis of randomly mistuned bladed disks". ASME Journal of Engineering for Gas Turbines and Power, 123 April , pp. 388-394.

[11] Griffin, J., and Hoosac, T., 1984. "Model development and statistical investigation of turbine blade mistuning". ASME Journal of Vibration, Acoustics, Stress, and Reliability in Design, 106 April , pp. 204-210.

[12] Sinha, A., and Chen, S., 1989. "A higher order to compute the statistics of forced response of a mistuned bladed disk assembly". Journal of Sound and Vibration, 2 (130), pp. 207-221.

[13] Yang, M.-T., and Griffin, J., 2001. "A reduced-order model of mistuning using a subset of nominal modes". ASME Journal of Engineering for Gas Turbines and Power, 123 (3) October, pp. 893-900.

[14] Castanier, M., Ottarson, G., and Pierre, C., 1997. “A reduced order modeling technique for mistuned bladed disks". ASME Journal of Vibration and Acoustics, 119 , pp. 439-447.

[15] Bladh, R., Castanier, M., and Pierre, C., 2001. "Component-mode-based reduced order modeling techniques for mistuned bladed disks-part 1: Theoretical models". ASME Journal of Engineering for Gas Turbines and Power, 123 (1) January , pp. 89-99.

[16] Petrov, E., Sanliturk, K., and Ewins, D., 2002. "A new method for dynamic analysis of mistuned bladed disks based on the exact relationship between tuned and mistuned systems". ASME Journal of Engineering for Gas Turbines and Power, 124 (3) July , pp. 586-597.

[17] Seinturier, E., Dupont, C., Berthillier, M., and Dumas, M., 2002. "A new aeroelastic model for mistuned bladed disks". In AIAA paper 2002-1533, 43rd AIAA/ASME/ASCE/AHS/ASC Structures, Structural Dynamics, and Materials Conference.

[18] Soize, C., 2000. "A nonparametric model of random uncertainties for reduced matrix models in structural dynamics". Probabilistic Engineering Mechanics, 15 (3), pp. 277-294.

[19] Soize, C., 2001. "Maximum entropy approach for modeling random uncertainties in transient elastodynamics.". Journal of the Acoustical Society of America, 109 (5) May , pp. 1979-1996.

[20] Soize, C., 2001. "Nonlinear dynamical systems with nonparametric model of random uncertainties.". E-journal from Resonance Publication (http://www.resonancepub.com), 1 (1) , pp. 1-38.

[21] Benfield, W., and Hruda, R., 1971. "Vibration analysis of structures by component mode substitution". AIAA Journal, 9 (7), pp. 1255-1261.

[22] Craig, R., and Bampton, M., 1968. "Coupling of substructures for dynamic analyses". AIAA Journal, 6 (7) July , pp. 1313-1319.

[23] Soize, C., 2003. "Uncertain dynamical systems in the medium-frequency range.". ASCE Journal of Engineering Mechanics, 129 (9), pp. 1017-1027.

[24] Soize, C., and Chebli, H., 2003. "Random uncertainties model in dynamic substructuring using a nonparametric probabilistic model.". ASCE Journal of Engineering Mechanics, 129 (4) , pp. 449-457.

[25] Capiez-Lernout, E., and Soize, C., 2003. "Probabilistic model of random uncertainties in structural dynamics for mistuned bladed-disks.". Mécanique et Industries, 4 (5), pp. 585-594. 